\title{
CALIDAD PERCIBIDA DE LA ATENCIÓN DE ENFERMERÍA POR PACIENTES HOSPITALIZADOS ${ }^{1}$
}

\section{PERCEIVED QUALITY OF NURSING CARE FOR HOSPITALIZED PATIENTS}

\author{
Yeis Miguel Borré Ortiz ${ }^{*}$ \\ Yolanda Vega Vega ${ }^{* *}$
}

\begin{abstract}
RESUMEN
El objetivo de la presente investigación fue determinar la calidad percibida de la atención de Enfermería por pacientes hospitalizados en una Institución de Servicios de Salud de Barranquilla (Colombia). Material y Método: se llevó a cabo un estudio descriptivo, de corte transversal, cuantitativo, en el que se encuestaron 158 pacientes a través de un cuestionario de calidad percibida, previo cumplimiento de criterios de inclusión. Los datos se tabularon y graficaron en Microsoft Excel, utilizando medidas de tendencia central, y frecuencias absolutas y relativas; guardando el rigor ético y metodológico correspondiente. Resultados: El 44\% fueron hombres y el $56 \%$ mujeres, con una media de 48.5 años. La calidad percibida de la atención de Enfermería es "Peor y mucho peor de lo que esperaba" en un 4\%, "Como me lo esperaba" en un 17\%, y "Mejor y mucho mejor de lo que me esperaba" en un 78\%. El 48.7\% estuvo "Muy Satisfecho", el 48.1\% "Satisfecho", y el 3\% "Poco y Nada Satisfecho". Conclusión: El 78\% de los pacientes evidenció altos niveles de calidad percibida de la atención de Enfermería; el 17\% modestos niveles de calidad percibida; y el $4 \%$ bajos niveles de calidad percibida. El $96.8 \%$ se sintió satisfecho y el 97\% recomendaría el hospital.
\end{abstract}

Palabras clave: Calidad de la atención de salud, atención de enfermería, satisfacción del paciente, hospitalización.

\begin{abstract}
The aim of this investigation was to determine the perceived quality of nursing care for patients hospitalized in an institution Health Services Barranquilla (Colombia). Method: A descriptive, cross-sectional, quantitative study, in which 158 patients were surveyed through a questionnaire of perceived quality, after fulfilling the inclusion criteria, was conducted. The data were tabulated and graphicated in Microsoft Excel, using central tendency measures and absolute and relative frequencies, keeping the appropriate ethical and methodological rigor. Results: $44 \%$ were male and $56 \%$ female, with a mean of 48.5 years old. The perceived quality of nursing care is "worse and much worse than I expected" by $4 \%$, "As I expected" by $17 \%$, and "Better, much better than I expected" by 78\%. The 48.7\% were "Very Satisfied", 48.1\% "Satisfied", and 3\% "Soon and Not Satisfied".

\footnotetext{
${ }^{1}$ Artículo de investigación derivado de la tesis de maestría: Calidad Percibida de la Atención de Enfermería por pacientes hospitalizados en Institución Prestadora de Servicios de Salud de Barranquilla 2013.

${ }^{*}$ Enfermero. Profesor Facultad de Ciencias de la Salud, Universidad Libre Seccional Barranquilla. Colombia. E-mail: yeismiguel@gmail.com

** Enfermera. Profesora asociada Facultad de Enfermería, Universidad Nacional de Colombia. Bogotá D.C. (Colombia). E-mail: yvegav@unal.edu.co
} 
Conclusion: $78 \%$ of patients showed high levels of perceived quality of nursing care, $17 \%$ modest levels of perceived quality, and $4 \%$ lower levels of perceived quality. The $96.8 \%$ was satisfied and $97 \%$ would recommend the hospital.

Key words: Quality of health care, nursing care, patient satisfaction, hospitalization.

Fecha recepción: 17/11/13. Fecha aceptación: 21/11/14.

\section{INTRODUCCIÓN}

La calidad y la satisfacción son dos conceptos que tienen gran interés y aplicación en la gerencia. Ambos han sido estudiados desde mediados del siglo XX. Sin embargo, hablar de calidad y satisfacción aún resulta complejo. La literatura evidencia múltiples definiciones que impiden conocer una definición universal (1). Existe consenso en que la calidad, con relación a la satisfacción, debe ser vista bajo dos tendencias: objetiva y subjetiva $(2,3)$. La primera, enfocada en el productor y la segunda, en el consumidor (2).

La calidad se ha definido como aquellas características que responden a las necesidades del cliente y la ausencia de deficiencias (4). Deming, afirmaba que solo podía definirse en función del sujeto que la juzga (5); y Crosby, la relacionaba con el cumplimiento de los requisitos del cliente (6). Todo lo anterior ha permitido el estudio de un concepto complejo y multifactorial denominado: "Calidad Percibida" (7), el cual consiste en una ecuación entre las expectativas y percepciones que determinan el nivel de satisfacción del usuario (7-10).

Figura 1. Modelo de Desconfirmación de Expectativas de Oliver

$$
\begin{aligned}
& P>E \Longleftrightarrow \text { Desconfirmación (+) } \Rightarrow \text { Altos Niveles de Calidad Percibida } \Longrightarrow \text { Mucha Satisfacción } \\
& \mathrm{P}=\mathrm{E} \Longleftrightarrow \text { Confirmación }(=) \Longleftrightarrow \text { Modestos Niveles de Calidad Percibida } \Longrightarrow \text { Satisfacción } \\
& \mathrm{P}<\mathrm{E} \Rightarrow \text { Desconfirmación (-) } \Longrightarrow \text { Bajos Niveles de Calidad Percibida } \Rightarrow \text { No Satisfacción }
\end{aligned}
$$

Fuente: Adaptación interpretativa elaborada por los autores, 2012.

Últimamente, las instituciones de salud han mostrado interés por mejorar la calidad de sus servicios. Una forma de hacerlo ha sido mediante la evaluación objetiva y subjetiva con el fin de lograr la satisfacción de expectativas del usuario (10), incluso sobrepasarlas $(11,12)$; dando importancia a la opinión del mismo (13). Convirtiéndose la satisfacción del usuario en un indicador esencial para la mejora continua de la calidad (14), y como instrumento para legitimar las diferentes reformas sanitarias (15). Motivo por el cual las instituciones de salud, posterior a la atención, aplican encuestas de satisfacción a sus usuarios; ya sea por medio físico, virtual o telefónico $(9,14,16)$, debido a que el usuario es quien tiene la oportunidad de juzgar, mediante su experiencia, la atención recibida (17).

En Colombia, con el establecimiento de la Ley 100 de 1993 y el Decreto 1011 de 2006, se introdujo el principio de calidad: bajo el cual se contempla la necesidad de crear mecanismos de control a los servicios de salud, 
para garantizar a los usuarios la calidad en la atención oportuna, personalizada, humanizada, integral y continua $(18,19)$.

Actualmente, con la reforma del Sistema de Salud mediante la Ley 1438 de 2011, se busca fortalecer la atención en salud a través del establecimiento de indicadores de evaluación que permitan medir el impacto de las actividades en las instituciones de salud, garantizar un adecuado acceso, oportunidad y calidad de los servicios a la población. Todo ello, desde una estrategia de Atención Primaria en Salud (APS), en donde el centro de atención sea el usuario (20).

Cabarcas et al. (21) sostienen que las instituciones de salud necesitan reemprender el tema de la calidad, puesto que su atención va dirigida a mantener el bienestar físico, mental y social de seres humanos. Atención proporcionada por personal de salud, dentro del cual se encuentra Enfermería; uno de los recursos humanos más importantes y con potencialidades para generar cambios en el cumplimiento de políticas de salud y en el logro de metas propuestas por las instituciones (22).

Durán et al. (23) y Correa (24), afirman que Enfermería es un recurso fundamental y el eje insustituible alrededor del cual gira la totalidad de la atención en salud, debido a que es el personal que permanece más tiempo y establece mayor contacto con el paciente durante la realización de procedimientos que se ven reflejados en la evaluación de resultados como parte de la atención integral (21, $25)$. Por consiguiente, la actividad de Enfermería responde a retos de equidad, fiabilidad, efectividad, buen trato, respeto, información, continuidad y confortabilidad; los cuales se constituyen en los condicionantes de la satisfacción del usuario (14).

Por tal razón, es importante que las acciones de Enfermería sean constantemente evaluadas y mejoradas (26). Sin embargo, para que el cuidado sea legítimo, íntegro, oportuno, continuo y efectivo, debe brindarse en las mejores condiciones, con los elementos disponibles, cumpliendo de manera total o parcial una necesidad o expectativa. Dando respuesta a las necesidades de bienestar del paciente, con el mejor recurso humano, material y aspecto técnico-científico de los que se dispone; buscando el máximo grado de satisfacción $(25,27)$.

La literatura evidencia la existencia de numerosos estudios sobre calidad percibida de la atención de Enfermería y satisfacción de los pacientes. No obstante, no todos muestran resultados buenos. En la mayoría se observa que la atención de Enfermería tiene significativas deficiencias $\mathrm{y}$, por tanto, debe mejorar (25, 28-36).

Esto indica que no todo está escrito con respecto a la calidad en la atención de Enfermería, ni los elementos para brindar cuidado con calidad son suficientes (37). La realidad evidencia que aún se presentan inconsistencias tales como: inadecuada entrega y recibo de turno, discontinuidad del cuidado, escasa atención a las necesidades de salud de los pacientes, aumento en las tasas de eventos adversos atribuidos a Enfermería, falta de comunicación entre el equipo interdisciplinario, escasez de información y orientación al paciente sobre los procedimientos a realizar, falta de atención al llamado, y más aún, cuando el paciente no llega a conocer el servicio en el que se encuentra hospitalizado y reconocer al personal de Enfermería que lo cuida, porque ni siquiera se presentan.

Todo lo anterior produce insatisfacción en el paciente. Situación que posteriormente se verá reflejada al rechazar la atención y no recomendar la institución.

Esta realidad se debe indagar porque Enfermería desempeña un rol indispensable dentro del equipo de salud, por ser el recurso humano de mayor número al ocupar el $40 \%$ y hasta el 60\% del personal de salud (38-40); es por ende, el más visible (41), sobre el cual recaen las miradas, y al que con facilidad juzgan los usuarios.

El modelo de la Fundación Europea para la Gestión de la Calidad (EFQM), recomien- 
da que la calidad debe medirse y evaluarse de forma sistemática $(16,42)$; por cuanto lo que se espera es conocer de forma permanente, el cómo se está ofreciendo la atención y en qué aspectos específicos habría que plantearse acciones de mejora continua.

En Barranquilla, el estudio de la calidad percibida es incipiente. Pocas investigaciones se han realizado en las instituciones de salud. Sin embargo, es común conocer la experiencia que han tenido las universidades en cuanto a la realización de diagnósticos situacionales, los cuales muestran que uno de los aspectos con mayor deficiencia es la atención de Enfermería. Por tanto, se evidencia la necesidad de que dicha atención se evalúe constantemente desde perspectivas objetivas y subjetivas.

El objetivo de la presente investigación fue determinar la calidad percibida de la atención de Enfermería por pacientes hospitalizados en una Institución de Servicios de Salud de Barranquilla.

\section{MATERIAL Y MÉTODO}

Se desarrolló un estudio descriptivo, de corte transversal, cuantitativo en el que se tuvo en cuenta toda la población que egresó diariamente de los servicios de hospitalización durante un trimestre del año 2011. Fueron excluidos aquellos pacientes que presentaron los siguientes criterios: menores de 18 años, discapacidad mental aparente y/o diagnosticada, alteraciones del lenguaje, alteración del estado de conciencia, sedación por medicamentos, y menos de dos días de hospitalización. 158 fue el total de pacientes que fueron encuestados durante el trimestre agosto, septiembre y octubre de 2011. El instrumento utilizado fue el cuestionario de calidad percibida (SERVQHOS-E) de Barragán y Manrique, el cual es una versión validada para Enfermería, tomada del SERVQHOS original de
Mira et al. (43). Dicha versión está dividida en tres partes: escala de aspectos tangibles e intangibles con 16 ítemes puntuados de 1 a 5 (escala Likert) que miden calidad percibida, datos de opinión y datos sociodemográficos. Además, posee confiabilidad alpha de Cronbach de 0.96 (44).

Para su aplicación en Barranquilla fue necesario realizar una prueba piloto con 32 participantes en la institución durante el mes de julio de 2011, guardando el rigor ético y metodológico, con el fin de corregir aspectos de lenguaje; debido a que se validó en una región de Colombia diferente en cultura.

Los resultados de la prueba piloto sugirieron modificaciones al cuestionario, en relación al lenguaje de algunos ítemes de opinión y datos sociodemográficos. Dichas modificaciones no alteraron de ninguna manera las propiedades psicométricas del cuestionario, debido a que los ítemes modificados no puntúan dentro de la escala, sino que son datos complementarios. En cuanto a la escala likert que mide aspectos tangibles e intangibles de la atención de Enfermería, no hubo necesidad de modificación porque se entendió bien en el contexto de estudio. Las modificaciones al cuestionario fueron necesarias para evitar interpretaciones erradas de las preguntas o el sesgo de no respuesta por falta de opción (45). Los encuestadores entrenados participaron en la aplicación y ajuste del instrumento, lo cual garantiza la estandarización en la recolección de datos.

La recolección de la información fue realizada por dos personas previamente entrenadas. Se identificaron los pacientes que cada día tenían orden de egreso (alta hospitalaria). Previa explicación de la investigación y su participación en ella, se le entregó a cada paciente un paquete sellado con el consentimiento informado y el cuestionario para su diligenciamiento. Se buscó la forma de que el paciente se encontrara en un ambiente privado, fuera de distracciones y en silencio; tanto en las habitaciones unipersonales como en 
las multipersonales. Una vez cada paciente terminaba el diligenciamiento de los documentos, éstos se introducían en un paquete tipo manila y delante de cada paciente era sellado, con el fin de preservar la confidencialidad de la información. Para garantizar el rigor ético y científico de la investigación, se obtuvo el aval del comité de ética de la Universidad Nacional de Colombia, la aprobación de los autores del instrumento para utilizarlo, y la autorización institucional para la recolección de la información. Se tuvieron en cuenta los parámetros establecidos en la Resolución 008430 de 1993 y las normas del Consejo de Organizaciones Internacionales de las Ciencias Médicas (CIOMS).

La información recolectada fue transcrita por el investigador para evitar sesgos en la consolidación de los datos. Se utilizó el programa Microsoft Excel para la organización, agrupación y procesamiento de los mismos.
Para el análisis de la información se utilizó análisis univariado mediante estadística descriptiva como: medidas de tendencia central, desviación estándar, frecuencias absolutas y relativas, y finalmente fueron graficadas con histogramas y diagrama de sectores.

\section{RESULTADOS}

El $44 \%$ fueron hombres y el $56 \%$ mujeres, con una media de 48.5 años (DE: 18.5). La Tabla 1 muestra los resultados obtenidos relacionados con: estado actual, estado civil y nivel de estudios alcanzado por la población estudiada.

El Gráfico 1 muestra el porcentaje de distribución de los pacientes encuestados de acuerdo a los diferentes servicios de hospitalización de la institución.

Tabla 1. Distribución porcentual de la población según variables sociodemográficas.

\begin{tabular}{lr}
\hline Variables sociodemográficas & FR \\
\hline Estado actual & \\
Pensionado & 0 \\
Desempleado & 31 \\
Empleado & 9 \\
Hogar & 46 \\
Estudiante & 8 \\
Trabajador Independiente & 6 \\
\hline Estado Civil & \\
Soltero(a) & 23 \\
Casado(a) & 32 \\
Viudo(a) & 8 \\
Separado(a) & 8 \\
Unión Libre & 29 \\
\hline Estudios & \\
Sin Estudios & 9 \\
Primarios & 44 \\
Bachillerato & 39 \\
Técnicos & 3 \\
Universitarios & 4 \\
\hline
\end{tabular}


Gráfico 1. Distribución de egreso por servicio de hospitalización.

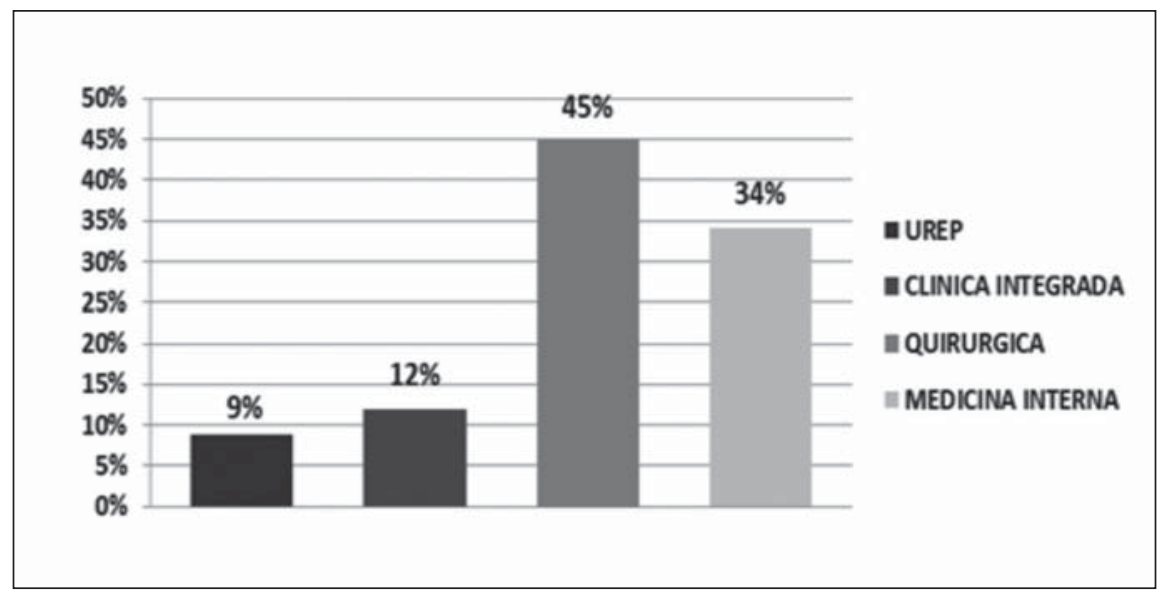

\section{Calidad Percibida}

Teniendo en cuenta que la calidad percibida es un fenómeno multifactorial y subjetivo, se decidió analizar su comportamiento por cada uno de los meses en los que se hizo la recolección de la información, y luego el comportamiento general en el trimestre, con el fin de identificar si existía variabilidad en el fenómeno estudiado. El análisis de ambos factores (tangibles e intangibles) permitió mostrar resultados de calidad percibida de forma individual y general. La agrupación de ambos factores por opciones de respuesta mostró que durante el trimestre hay una tendencia marcada hacia las opciones 3,4 y 5 (Gráfico 2), lo cual indica que para la mayoría de los pacientes, las expectativas previas fueron superadas por las percepciones obtenidas durante la atención de Enfermería. A partir de este hallazgo, es importante resaltar que las opciones de respuesta 1 y 2 carecieron de significancia para develar niveles bajos de calidad percibida.

Gráfico 2. Relación del comportamiento de aspectos tangibles e intangibles en el trimestre.

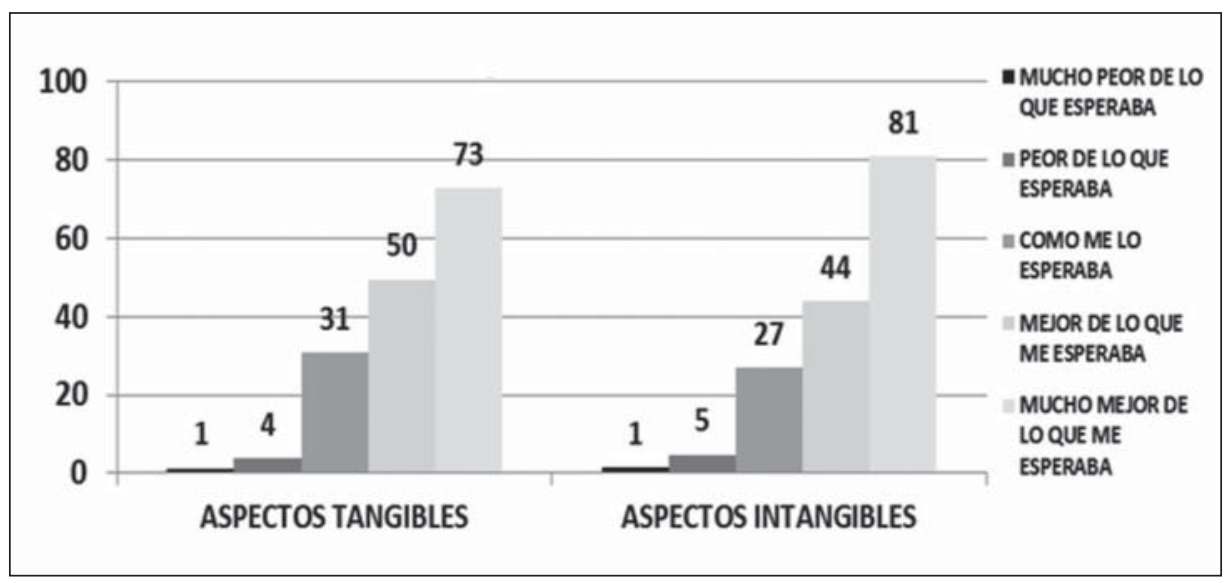


En los gráficos 3 y 4 se observa que durante el mes de septiembre el tipo de respuesta "Como me lo esperaba" sobresalió de forma significativa frente a los otros dos meses. No obstante, en los meses agosto y octubre sobresale el tipo de respuesta "Mucho mejor de lo que me esperaba".

Gráfico 3. Aspéctos tangibles de calidad percibida.

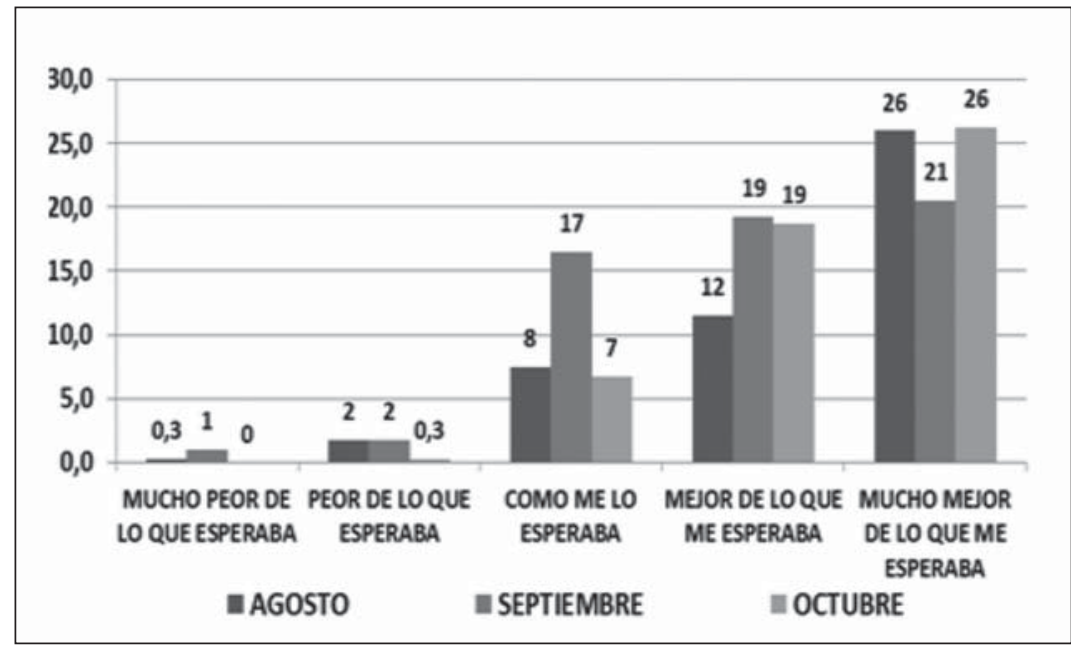

Gráfico 4. Aspectos intangibles de calidad percibida.

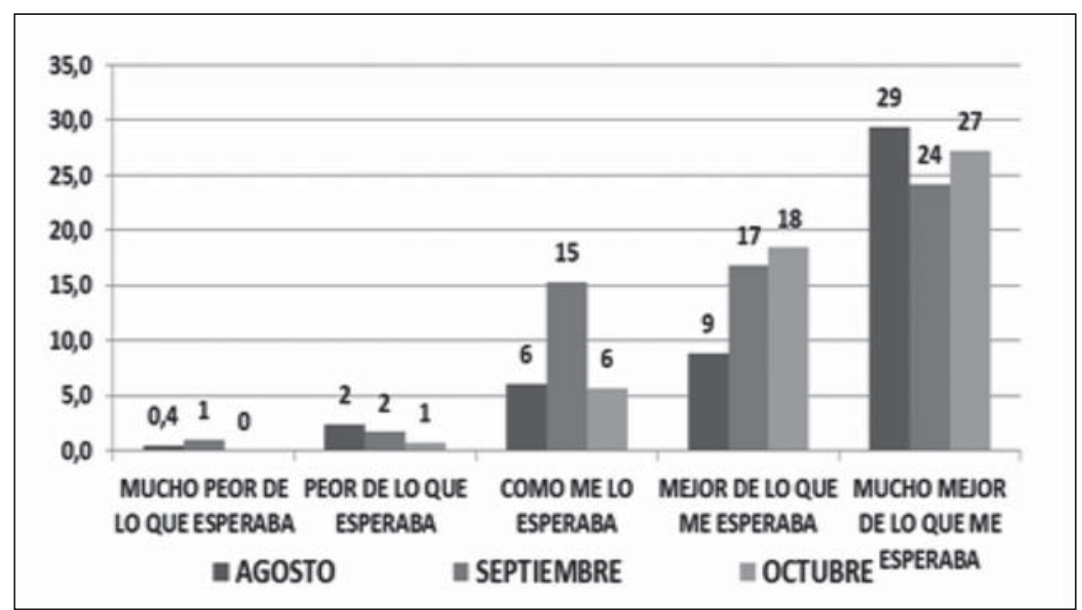

Mostrando que los pacientes egresados en agosto y octubre manifestaron mayores niveles de calidad percibida que los egresados en septiembre. Finalmente, las opciones de respuesta fueron agrupadas en tres grandes opciones (Gráfico 5).

De esta manera, la calidad percibida fue "Mucho peor de lo que esperaban" y "Peor de lo que esperaban" en un 4\%, "Como lo esperaban" en un $17 \%, y$ "Mucho mejor de lo que 
Gráfico 5. Calidad percibida de la atención de enfermería en el trimestre.

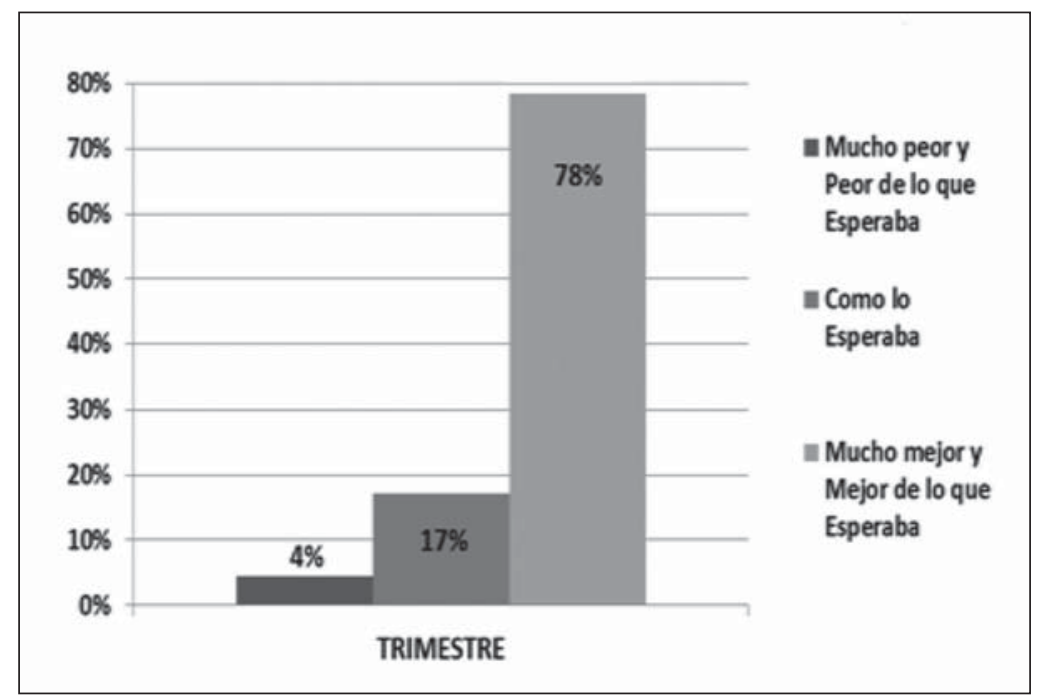

esperaban" y "Mejor de lo que esperaban" en un $78 \%$. Corroborando que los pacientes hospitalizados percibieron altos niveles de calidad en la atención de Enfermería.

\section{Datos de Opinión}

El $96.8 \%$ de los pacientes encuestados se encontró satisfecho con la atención de Enfermería (48,7\% muy satisfecho, $48,1 \%$ satisfecho), hallazgo que guarda relación con los altos niveles de calidad percibida en los servicios.

Por otra parte, el 97\% de los encuestados manifestó que recomendaría el hospital, sin dudarlo, a otras personas. Sólo el 3\% tiene dudas y nunca lo haría. Resultado que guarda relación con el nivel de satisfacción global.

Con respecto al resto de las preguntas de opinión, el 90\% afirmó que el personal de Enfermería no le hizo ningún tipo de procedimiento sin su permiso, mientras que el $10 \%$ aludió que sí. Este hallazgo refleja que el equipo de Enfermería en su gran mayoría sí informa al paciente sobre los procedimientos a realizar, pero debe hacerlo siempre y más seguido con cada uno en particular, teniendo en cuenta la individualidad del cuidado.
El $86 \%$ de los pacientes considera que estuvo hospitalizado el tiempo necesario para su recuperación, mientras que el 14\% restante manifestó haber estado menos o más del tiempo necesario en la institución. El 68\% dijo que no conocía el nombre de la enfermera que lo atendía en cada turno, mientras que el $32 \%$ dijo que sí.

El $83 \%$ aceptó que ha recibido suficiente información sobre su estado de salud, pero llama la atención que hay un $17 \%$ que se consideró no informado lo suficientemente por parte del personal de Enfermería. Aspecto que guarda relación directa con la pregunta acerca del conocimiento que tenía el paciente sobre el servicio en el que se encontraba internado. 50 de los 158 pacientes encuestados tenía idea de ello, mientras que los 108 restantes no. Lo cual indica que los pacientes no están del todo bien informados.

Según el tipo de ingreso al hospital, se encontró que el 48\% ingresó remitido de otra institución; esto se debe a que la institución centro de estudio es una institución de cuarto nivel y cuenta con la mayoría de los servicios de atención básica y especializada. El $29 \%$ ingresó de forma programada a través de la consulta externa y/o especializada, y el 
$23 \%$ restante ingresó por urgencias 2 . El $90 \%$ de los pacientes encuestados estuvo internado una sola vez en la institución, y sólo el $10 \%$ lo estuvo por más de dos veces durante ese mismo año, lo cual indica que la mayoría de los pacientes vivió por primera vez la experiencia de la atención de Enfermería en los servicios de hospitalización de la institución.

\section{DISCUSIÓN Y CONCLUSIÓN}

El cuestionario SERVQHOS-E es de reciente validación en Colombia (44) y éste es uno de los primeros estudios que lo utiliza como instrumento para la recolección de la información. Por tanto, es imperiosa la necesidad de comparar los resultados encontrados con los hallados en otros estudios que han utilizado cuestionarios diferentes.

Aunque algunos autores afirmen que las variables sociodemográficas como: sexo, nivel de estudios, ocupación, estado civil, estado de salud, experiencias pasadas con el personal de salud, personalidad del paciente, estilos de vida, edad, creencias y vivencias, influyen en el nivel de calidad percibida y la satisfacción de los pacientes (46-49); en el presente estudio no se encontró dicha influencia. Quizás, en estudios correlacionales posteriores pueda confirmarse tales hallazgos. No obstante, los resultados encontrados guardan coherencia con lo hallado en estudios revisados $(50,51)$.

La teoría de la Desconfirmación de Expectativas de Oliver y los postulados de Parasuraman et al. (52) plantean que la calidad percibida es una ecuación entre las expectativas previas que tiene un usuario antes de recibir

\footnotetext{
${ }^{2}$ Cabe mencionar que la institución no cuenta con el servicio de urgencias propiamente dicho, sino que existe el servicio de UREP (Unidad de Recepción y Evaluación de Pacientes), el cual se incluyó porque posee 22 camas en las cuales se internan pacientes por más de dos días y cuyos egresos son reportados dentro del censo estadístico como hospitalización. Además, este servicio está preparado para recibir pacientes que ingresen con alguna urgencia vital.
}

un servicio y su percepción posterior. Por tanto, el resultado final entre estos dos elementos da como resultado el nivel de satisfacción del usuario. En este sentido, aunque los resultados de calidad percibida por mes revelaron que la variabilidad del fenómeno es notable, y que cambia dependiendo los días de estancia, el tipo de pacientes y la percepción de cada uno en particular; en todo caso, fue percibida como buena con valores superiores. Esto fue corroborado cuando se hizo el análisis por todo el trimestre. Resultado similar al hallado por Utrilla ${ }^{3}$, quien observó que los usuarios percibieron calidad de atención de Enfermería favorable en un $84 \%$ y medianamente favorable en un $16 \%$; mientras que Quispe ${ }^{4}$ revela que la opinión del paciente sobre la calidad de atención de la enfermera es medianamente favorable y favorable en un $70 \%$ y desfavorable en un $30 \%$.

Otros estudios muestran resultados diferentes a los hallados en el presente. Torres y Rivas (50) afirman que la percepción del paciente hospitalizado respecto a la atención proporcionada por Enfermería es buena en un $97 \%$ y regular en un 3\%. Romero y Durand $^{5}$ muestran que la percepción del paciente es desfavorable y medianamente desfavorable en un $56.67 \%$ y favorable y medianamente favorable en un $43.33 \%$, indicando que las categorías negativas están por encima de las categorías positivas de la atención, y que además, están dadas por la relación interpersonal y actividades propias del proceso de atención. Aunque Puebla et al. (25) expo-

\footnotetext{
${ }^{3}$ Utrilla N. Percepción que tiene el usuario externo acerca de la calidad de atención de enfermería en el servicio de la unidad de cuidados intermedios en el Hospital Nacional Daniel Alcides Carrión [Tesis]. Lima: Universidad Nacional Mayor de San Marcos; 2009.

${ }^{4}$ Quispe AM. Opinión que tienen los usuarios del servicio de medicina general sobre la calidad de la atención de la enfermera en el Hospital Nacional Arzobispo Loayza [Tesis de Licenciatura]. Lima: Universidad Nacional Mayor de San Marcos; 2005.

${ }^{5}$ Romero LC, Durand J. Percepción del paciente acerca de la calidad de atención que brinda la enfermera en el servicio de medicina en el Hospital Nacional Daniel Alcides Carrión [Tesis de Licenciatura]. Lima: Universidad Nacional Mayor de San Marcos; 2008.
} 
nen que la calidad de la atención que ofrece el personal de Enfermería se debe reflejar en la satisfacción del paciente que la recibe, otros afirman que para medir correctamente dicha calidad no solamente se debe tener en cuenta la subjetividad del paciente, sino también aspectos objetivos presentes en el servicio (50). El cuestionario SERVQHOS-E está diseñado para medir ambos aspectos, pero desde perspectivas: tangible, intangible y la satisfacción del paciente; siendo la satisfacción uno de los métodos mediante los cuales se puede obtener información acerca de cómo están los resultados de la atención en salud $(14,53)$.

Según Barragán y Manrique (44) la satisfacción del usuario obedece más a la funcionalidad del servicio. Es decir, a aspectos tangibles e intangibles dentro del mismo; mientras que la calidad percibida apunta a una evaluación global y es pertinente puesto que permite la toma de decisiones gerenciales y condiciona la atención.

Los aspectos tangibles son aquellos que utiliza Enfermería para facilitar la atención; dentro de ellos: el espacio físico, la tecnología y el recurso material. Los aspectos intangibles hacen referencia a las cualidades propias que el personal de Enfermería manifiesta durante el proceso de atención, dentro de ellas: la empatía, el interés, la cortesía, la confianza, la puntualidad, la rapidez, el trato personalizado y la capacidad profesional (44).

En el presente estudio se encontró que los aspectos tangibles e intangibles mejor valorados fueron: la apariencia del personal, el sistema de señalización en los servicios, la tecnología, la puntualidad, la confianza, la amabilidad, la preparación académica, el trato personalizado y la capacidad para comprender las necesidades del paciente. También se valoraron de forma medianamente favorable: la información proporcionada, el interés por cumplir lo que prometen y la rapidez en la atención. Los aspectos peor valorados fueron: la apariencia y comodidad de las habitaciones, el interés en la resolución de problemas, el tiempo de espera y la actitud de disposición. Resultados que están por debajo de los hallados por Cabarcas et al. (21), quienes encontraron que la satisfacción relacionada con la presencia de elementos tangibles en la actividad de Enfermería alcanzó 100\% en apariencia pulcra del personal y limpieza de elementos materiales, y $93 \%$ en cuanto a la apariencia moderna de los equipos.

Algo totalmente contrario a lo hallado por González et al. (29), quienes observaron que los ítemes peor valorados fueron los incluidos en la dimensión de aspectos tangibles, indicando que el ambiente de la institución era poco acogedor.

Donabedian, el primer y mayor exponente de la teoría de evaluación de la calidad en salud, otorgó a los aspectos tangibles un valor agregado determinante para medir calidad asistencial total. Sin embargo, en sus postulados expresaba que las instituciones de salud, además de mantener una buena estructura, un adecuado proceso y unos resultados impactantes, no debían restar interés por buscar la satisfacción del paciente, porque el fin último de la calidad es tratar de conocer lo que percibe y exige el paciente para después actuar en fin común $(49,53)$.

El estudio de Pujiula et al. (49) reveló que los pacientes expresaron altos niveles de satisfacción. Sin embargo, entre los aspectos peor valorados figuran el espacio físico y la calidad de la información, debido a que pocos centros hospitalarios disponen de estructuras atractivas y confortables. Todo ello, unido a la frecuente pérdida de la intimidad a causa de la propia actividad hospitalaria, generando aún más insatisfacción.

En el presente estudio el $96.8 \%$ de los pacientes encuestados estuvo satisfecho y muy satisfecho con la atención de Enfermería. Resultado que está por encima del expresado por Govín et al. (54), quienes encontraron un nivel de satisfacción del $80,8 \%$ e insatisfacción del 19,2\%. Cabarcas et al. (21) hallaron que la satisfacción del paciente fue de $85 \%$. Verdugo et al. (55) encontraron que el 92.5\% de las pacientes con cáncer mamario 
estuvieron satisfechas. González et al. (29) manifiestan haber encontrado una satisfacción del usuario razonablemente alta. Sin embargo, existen estudios con resultados más altos que el presente (56). En cuanto al estar poco y nada satisfecho con la atención de Enfermería, los resultados son coherentes con lo encontrado por Mira et al. (48), quienes evidencian que, en su estudio, solamente el 3\% se declaró muy insatisfecho.

Los resultados de satisfacción global y recomendación del hospital encontrados en el presente estudio corroboran lo explicado por Barragán y Manrique (44), quienes demostraron que existe alta correlación entre ambos ítemes. No obstante, es totalmente contrario al encontrado por Mira et al. (48) quienes expresan que el resultado obtenido en la variable "Recomendar el hospital" fue inesperado, debido a que la cifra de pacientes que no recomendaría el hospital donde estuvieron ingresados fue elevada.

Este estudio encontró que la calidad percibida de la atención de Enfermería, habiendo sido evaluada mes a mes durante un trimestre, mostró una tendencia buena en un $95 \%$. Resultado que guarda coherencia con otros estudios que evidencian porcentajes de calidad percibida dentro del intervalo de 60 a $100 \%$. Además, dicha percepción está relacionada directamente con el nivel de satisfacción global de los pacientes y el hecho de recomendar el hospital. Hallazgo que, según Barragán et al. $(57,58)$ y la teoría de Desconfirmación de expectativas de Oliver, indica altos niveles de calidad percibida en la institución.

No se encontró ninguna relación directamente proporcional entre los aspectos sociodemográficos con respecto a la calidad percibida y la satisfacción de los usuarios, debido al alcance del estudio. Sin embargo, en estudios correlacionales posteriores en los que se utilice el mismo cuestionario, podría analizarse si las variables sociodemográficas influyen de manera significativa en dicha percepción.
Es importante señalar que el SERVQHOS original es un instrumento que ha sido utilizado en muchas investigaciones; mientras que el SERVQHOS-E es un cuestionario validado recientemente en Colombia. Por tanto, los hallazgos del presente estudio permiten mostrar la eficacia del mismo en la medición del fenómeno, pero también permite identificar aspectos relevantes de la atención de Enfermería desde el punto de vista del paciente.

Este es uno de los primeros estudios, a nivel nacional, que utiliza el instrumento SERVQHOS-E, razón por la cual es necesario que se sigan realizando investigaciones en otros contextos para corroborar la confiabilidad del instrumento. No obstante, se recomienda previa validez aparente debido a las diferentes culturas.

Agradecimientos: Los autores agradecen a la Doctora Cristina García-Vivar de la Universidad de Navarra (España) y a la Magíster Karla Mijangos-Fuentes de la Universidad de la Sierra Sur (México), por la revisión y aportes especiales al presente manuscrito.

\section{REFERENCIAS}

1. Colmenares O, Saavedra JL. Aproximación teórica de los modelos conceptuales de la calidad del servicio. Técnica Administrativa [Internet]. 2007 [citado 5 julio 2013]; 6(4). Disponible en: http://www. cyta.com.ar/ta0604/v6n4a2.htm

2. Duque EJ. Revisión del concepto de calidad del servicio y sus modelos de medición. Rev. Innovar 2005; 15(25): 64-80.

3. Huber D. Mejora en la calidad y control de riesgos. En: Huber D. Liderazgo y administración en enfermería. México D.F.: McGraw-Hill; 1999. p. 467-90.

4. Llinás A. Evaluación de la calidad de la atención en salud, un primer paso para la reforma del sistema. Salud Uninorte 
2010; 26(1): 143-54.

5. Deming E. Calidad, productividad y competitividad: La salida de la crisis. Madrid: Díaz de Santos; 1989.

6. Crosby P. La Calidad no cuesta: el arte de cerciorarse de la calidad. México: McGraw Hill; 1987.

7. Medina M, Medina E. Análisis de la calidad percibida en usuarios/as de servicios sociales comunitarios. Rev Serv Soc. 2011; 50(1): 85-100.

8. Velandia F, Ardón N, Jara MI. Satisfacción y calidad: análisis de la equivalencia o no de los términos. Rev Gerenc Polit Salud. 2007; 6(13): 139-68.

9. Betancourt Y, Mayo JC. La evaluación de la calidad de servicio. Contribuciones a la Economía [Internet]. Enero 2010 [citado 20 julio 2013]: [Aprox. 1 p.]. Disponible en: http://www.eumed.net/ce/2010a/ bama.htm

10. Evans JR, Lindsay WM. Administración y control de la calidad. $4^{\mathrm{a}}$ ed. México: Internacional Thompson; 2000.

11. Sepúlveda GJ, Rojas LA, Cárdenas OL, Guevara E, Castro AM. Estudio piloto de la validación del cuestionario 'CARE-Q' en versión al español en población colombiana. Rev. colomb. Enferm. 2009; 4(4): 13-22.

12. Lozada M, Rodríguez A. Calidad del servicio de salud: Una revisión a la literatura desde la perspectiva del marketing. Cuad. Adm. 2007; 20(34): 237-58.

13. Ardila A, Garay G. Caracterización de la calidad percibida de la prestación de servicios de salud según los usuarios de Unisalud [Tesis de Maestría]. Bogotá D.C: Universidad Nacional de Colombia; 2006.

14. Jiménez M, Ortega VMC, Cruz AG, Cruz CM. Satisfacción del usuario como indicador de calidad. Rev Mex Enferm Cardiol. 2003; 11(2): 58-65.

15. Caminal J. La medida de la satisfacción: un instrumento de participación de la población en la mejora de la calidad de los servicios sanitarios. Rev Calid Asist.
2001; 16: 276-79.

16. Mira JJ, Aranaz J, Lorenzo S, Rodríguez J, Moyano S. Evolución de la calidad percibida por los pacientes en dos hospitales públicos. Psicotherma. 2001; 13(4): 58185.

17. Lenis CA. Calidad del Cuidado de Enfermería percibida por pacientes hospitalizados en una Institución de III nivel de Barranquilla [Tesis de Maestría]. Bogotá D.C.: Universidad Nacional de Colombia; 2013.

18. Ley 100 de 1993. Por la cual se crea el Sistema de Seguridad Social Integral y se dictan otras disposiciones. Congreso de la República de Colombia. Diario Oficial $\mathrm{N}^{\circ} 41.148$ (Diciembre 23, 1993).

19. Decreto 1011 de 2006. Por el cual se establece el Sistema Obligatorio de Garantía de Calidad de la Atención de Salud del Sistema General de Seguridad Social en Salud. Presidencia de la República de Colombia. Diario Oficial No 46.230 (Abril 3, 2006).

20. Ley 1438 de 2011. Por medio de la cual se reforma el Sistema General de Seguridad Social en Salud y se dictan otras disposiciones. Congreso de la República de Colombia. Diario Oficial No 47.957 (Enero 19, 2011).

21. Cabarcas IN, Montes A, Ortega G. Satisfacción del paciente hospitalizado con la atención de Enfermería en una institución de salud de la ciudad de Cartagena, Colombia. Actual. enferm. 2009; 12(1): 8-11.

22. Zárate R. La gestión del cuidado de enfermería. Index Enferm 2004; 13(44-45): 42-6.

23. Durán E, Cuervo MP, Echeverri S. Desarrollo de la Enfermería en Colombia. Acto de reconocimiento. Actual. enferm. 2004; 7(1): 26-31.

24. Correa P. Las colombianas, entre las más buscadas: Enfermeras en plan de fuga. El Espectador.com [Internet]. 18 octubre 2008 [citado 5 julio 2013]. Disponible 
en: http://www.elespectador.com/impreso/articuloimpreso84749-enfermeras-plan-de-fuga

25. Puebla DC, Ramírez A, Ramos P, Moreno MT. Percepción del paciente de la atención otorgada por el personal de Enfermería. Rev Enferm Inst Mex Seguro Soc. 2009; 17(2): 97-102.

26. Gaviria DL. La Evaluación del cuidado de Enfermería: un compromiso disciplinar. Invest Educ Enferm. 2009; 27(1): 24-33.

27. Bernal D, Garzón N. Eventos adversos durante la atención de enfermería en unidades de cuidados intensivos [trabajo de grado]. Bogotá D.C: Pontificia Universidad Javeriana; 2008.

28. Schmidt LA. Patients' perceptions of nursing care in the hospital setting. J Adv Nurs. 2003; 44(4): 393-99.

29. González MA, Padin S, Ramón E. Satisfacción del paciente con la atención de enfermería. Enferm Clin. 2005; 15(3): 147-55.

30. Ariza C, Daza R. Sobre calidad del cuidado de enfermería al paciente hospitalizado en un hospital de tercer nivel en Bogotá. Actual. enferm. 2005; 8(3): 8-14.

31. Ponce J, Reyes H, Ponce G. Satisfacción laboral y calidad de atención de enfermería en una unidad médica de alta especialidad. Rev Enferm Inst Mex Seguro Soc. 2006; 14(2): 65-73.

32. Samira M, Gj Q, Tabish S, Samiya M, Riyas R. Patient's perception of nursing care at a large teaching Hospital in India. Int J Health Sci (Qassim). 2008; 2(2): 92-100.

33. Zhao SH, Akkadechanunt T, Xue XL. Quality nursing care as perceived by nurses and patients in a Chinese hospital. J Clin Nurs. 2009; 18(12): 1722-28.

34. Nashrath M, Akkadechanunt T, Chontawan R. Perceived nursing service quality in a tertiary care hospital, Maldives. Nurs Health Sci. 2011; 13(4): 495-501.

35. Kobayashi H, Takemura Y, Kanda K. Patient perception of nursing service quality; an applied model of Donabedian's structure-process-outcome approach theory. Scand J Caring Sci. 2011; 25(3): 419-25.

36. Momani AL, Korashy HA. Patient experience of nursing quality in a teaching hospital in Saudi Arabia. Iran J Public Health. 2012; 41(8): 42-9.

37. Machucca D. Calidad o excelencia "una preocupación de la gerencia”. Rev Cienc y Cuid. 2006; 3(3): 140-46.

38. Parra M. Perfil epidemiológico del multiempleo en el personal de enfermería que labora en el Hospital Universitario de Pediatría Dr. "Agustín Zubillaga" Estado Lara [trabajo de grado]. México D.F.: Universidad Centroccidental Lisandro Alvarado; 2008.

39. Vega Y. La administración que se enseña en el pregrado de enfermería. av. enferm. 2012; III(1): 95-06.

40. Malvárez S. La regulación de la práctica y la educación en enfermería. Nuevos determinantes [Internet]. En: Actas del XIII CURSO OPS/OMS-CIESS. Legislación de Salud: La regulación de la práctica profesional en salud. México DF: 4-8 septiembre; 2006. [citado 5 julio 2013]. Disponible en: http://www.paho.org/ spanish/dpm/shd/hp/hp-xiii-taller06pres-malvarez.pdf

41. Vega Y. Introducción de la asignatura Modelos Administrativos en Enfermería [CD ROOM]. Bogotá D.C: Universidad Nacional de Colombia; 2010.

42. Arcelay A, Lorenzo S, Bacigalupe M, Mira JJ, Palacio F, Ignacio E, et al. Adaptación de un modelo de gestión de calidad total al sector sanitario. Rev Calid Asist. 2000; 15(3): 184-91.

43. Mira JJ, Aranaz J, Rodríguez M, Buil AA, Castell M, Vitaller J. SERVQHOS: Un cuestionario para evaluar calidad percibida de la asistencia hospitalaria. Med Prev. 1998; 4: 12-8.

44. Barragán J, Manrique FG. Validez y confiabilidad del SERVQHOS para enfermería en Boyacá, Colombia. av. enferm. 
2010; 28(2): 48-61.

45. Borré YM, Lenis-Victoria C, González-Ruiz G. Utilidad del cuestionario SERVQHOS-E para medir calidad percibida de la atención de enfermería. Rev CES Salud Pública. 2014; 5(2): 127-36.

46. Akin S, Erdogan S. The turkish version of the newcastle satisfaction with nursing care scale used on medical and surgical patients. J Clin Nurs. 2007; 16(4): 646-53.

47. Torres CC. Percepción de la calidad del cuidado de enfermería en pacientes hospitalizados. av. enferm. 2010; 28(2): 9810.

48. Mira JJ, Buil J, Aranaz J, Vitaller J, Lorenzo S, Ignacio E, et al. ¿Qué opinan los pacientes de los hospitales públicos? Análisis de los niveles de calidad percibida en cinco hospitales. Gac Sanit. 2000; 14(4): 291-93.

49. Pujiula J, Suñer R, Puigdemont M, Grau A, Bertrán C, Hortal G, et al. La satisfacción de los pacientes hospitalizados como indicador de la calidad asistencial. Enferm Clin. 2006; 16(1): 19-26.

50. Torres C, Rivas JG. Percepción del paciente hospitalizado sobre el trato digno proporcionado por una enfermera-un enfermero. Rev Mex Enferm Cardiol. 2010; 18(3): 60-4.

51. Christoph R. Determinación de la calidad de la atención del profesional de enfermería en servicios de hospitalización de las instituciones prestadoras de salud especializadas en salud mental y psiquiatría de la ciudad de Barranquilla [Tesis de
Maestría]. Bogotá D.C: Universidad Nacional de Colombia; 2006.

52. Parasuraman A, Zeithaml V, Berry L. SERVQUAL: A multiple-item scale for measuring consumer perceptions of Service Quality. J Retailing. 1988; 64: 12-40.

53. Donabedian A. The quality of care. How Can It Be Assessed? JAMA. 1988; 260(12): 1743-48.

54. Govín J, Torres M, Regalado D. Calidad de la atención de enfermería. Rev Cien. Méd La Habana [Internet]. 2002 [citado 19 junio 2013]; 8(1). Disponible en: http://revcmhabana.sld.cu/index.php/ rcmh/article/view/90/157

55. Verdugo A, Esparza R, Magaña A, Arizona $M$. Factores que influyen en la calidad de atención de enfermería en pacientes con carcinoma mamario. Rev Enferm Inst Mex Seguro Soc. 2011; 19(1): 15-9.

56. Carrillo M, Martínez LE, Mendoza JR, Sánchez A, Yáñez R, Rivera RV, et al. Cumplimiento del indicador de calidad trato digno proporcionado por el personal de enfermería. Rev Enferm Inst Mex Seguro Soc. 2009; 17(3): 139-42.

57. Barragán JA, Pedraza YD, Rincón MR. Calidad percibida de la atención de enfermería por usuarios hospitalizados, Tunja, 2011. Rev. Fac. Nac. Salud Pública. 2013; 31(2): 187-93.

58. Barragán JA, Moreno CM. Calidad percibida por usuarios de enfermería en tres hospitales públicos. Enferm. glob. 2013; 12(1): 217-30. 